\title{
Data-Driven Distributed Reactive Power Sharing in Microgrids
}

\author{
Seyed Sohail Madani and Alireza Karimi
}

\begin{abstract}
In this paper, reactive power sharing for Photovoltaic (PV) units in islanded microgrids has been formulated as a robust control design problem and is solved using convex optimization method. In addition to reactive power sharing, the disturbance rejection for voltage and active power have been formulated using infinity-norm constraints on the sensitivity functions and considered in the design. The proposed method uses only the measurement data of the power system with no need for a parametric model of the power grid equipment. The size of the problem is independent of the order of the plant which makes it applicable to power systems including a high number of buses and equipment such as synchronous generators, batteries and inverters. In the proposed method, the communication system can be considered in the control design process for centralized, distributed and decentralized structures. The proposed method has been validated through simulation of a microgrid encompassing synchronous generator, switching inverters and storage system. The results show that this method has successfully shared reactive power among different PV units while providing disturbance rejection for voltage and active power.
\end{abstract}

\section{INTRODUCTION}

Global warming concerns has led to increase the share of renewable energy resources in electrcity generation. Except for hydro energy plants, most of renewable Distributed Generation units (DGs) such as PVs and the wind turbines are connected to the power grid using power-electronic converters. Since the renewable resources are intermittent, the ratings for different parts of the system are designed for maximum power, while the system usually does not operate in its full capacity. The priority for using the capacity of the equipment is with active power. However, the spare capacity of the converters can be used to provide ancillary services to the grid including reactive power compensation.

Traditionally, the reactive power in the distribution grid is provided by constant or switchable capacitor banks. Since the constant capacitors inject almost a constant reactive power, they may cause overvoltage when the system is not fully loaded. In switchable capacitor banks, the number of the possible steps are limited and causes high inrush current, over-voltages and harmonics [1]. As another solution, Static Var Compensators (SVCs) have been proposed to compensate the reactive power in distribution systems [2]-[4]. However, high harmonic injection and very low bandwidth are reported in industrial types of SVCs [3]. Distribution

This work is supported in part by the Swiss National Science Foundation under Grant 200021_172828 and by Swiss Federal Commission for Innovation and Technology within the SCCER-FURIES. (Corresponding author: Alireza Karimi.)

S. S. Madani and A. Karimi are with the Laboratoire d' Automatique, École Polytechnique Fédérale de Lausanne, 1015 Lausanne, Switzerland (e-mail: sohail.madani@epfl.ch; alireza.karimi@epfl.ch).
STATic synchronous COMpensator (D-STATCOM) [5], [6] improves the power quality and supports reactive power with high bandwidth. However, due to high investment costs, the applications are limited and the size of this equipment should be selected conservatively [7].

Instead of adding equipment, the spare capacity of power electronic converters of DGs can be used to compensate for reactive power [8]. However, sharing the reactive power among different DGs with the aim of avoiding converter overload is a challenging control problem. Different methods have been proposed for control of reactive power and sharing this power among DGs. A group of proposed methods are based on the idea of droop control [9], [10]. Although these methods are simple and easy to implement, the closedloop stability is not generally guaranteed. A sliding mode controller has been proposed in [11] for reactive power control of wind turbines. In [12], high-level optimal reactive power control has been proposed assuming each DGs can regulate the injected power to the grid. In [13], the stability boundaries of a wind power plant including a STATCOM and controlled by PI controller as voltage controllers have been assessed with the aim to damp the low-frequency reactive power oscillations. An adaptive control method for a wind turbine has been proposed in [14] for reactive power compensation while guaranteeing performance and boundedness of the signals. However, these methods need parametric models which are usually hard to achieve in power systems. In [15], a non-linear state feedback controller using communication system has been proposed in order to share the reactive power among different inverters in a distributed way. In [16], the reactive power has been controlled in order to be in maximum distance from the voltage bifurcation point to avoid the voltage collapse. However, the couplings in the power-flow equation have not been considered, which can be significant in distribution systems.

In this paper, a data-driven controller design method for the reactive power sharing problem is proposed. The reactive power sharing and other control performance are formulated using the infinity-norm bounds on input and output sensitivity functions. Then, the problem is written in the concaveconvex form and the concave part is linearized around an initial controller. Finally, the control problem is converted to a convex optimization problem with linear matrix inequalities (LMIs). In this method, there is no need for a parametric model of the power system and only measurement data is used in the design process. Moreover, there is no need for any assumption on the decoupling of active power from voltage and reactive power from frequency in this method, which makes it applicable to different distribution as well 
as transmission systems. Another significant advantage of this method is the capability of designing the controller for centralized, distributed or decentralized structures based on the availability of communication infrastructure.

The rest of the paper is organised as follows: In Section II, the data-driven controller design method has been described. In Section III, the reactive power sharing problem for PV units in power grids has been formulated as a set of LMIs. In Section IV, a controller has been designed for the case-study grid and its performance has been validated in simulation. Finally, the conclusions are presented in SectionV.

\section{Controller Design}

The design method in this paper is based on the frequency response of the system. If the parametric model is available the frequency response can be directly calculated. Since the parameters of the power system are not usually easy to extract, the frequency response is computed using the measurement data. Because the measurement data in a short time usually is not rich enough, adding an excitation signal can increase the accuracy of the frequency response. In this paper, a Pseudo-Random Binary Sequence (PRBS) has been used as the external excitation. Using the Fourier transform, an $m$-input $/ n$-output frequency response model $G\left(e^{j \omega}\right) \in$ $\mathbb{C}^{n \times m}$ around the operating point is calculated.

\section{A. Controller Structure}

The general structure of the controller is $K=X Y^{-1}$ where $X$ and $Y$ are matrix polynomials in $z$ (or in $s$ for continuous-time controllers). For sake of simplicity in notation, the argument of $e^{j \omega}, z$ or $s$ are omitted and will only be reiterated when it deemed necessary. This controller can be designed in centralized, distributed or decentralized structures based on the available communication links. If the communication link is not available between two points the corresponding element in the controller is fixed to zero.

\section{B. Sensitivity Functions}

The control design problem formulation in this paper is based on the method proposed in [17]. The goal is to formulate the control design problem in the form of a convex optimization problem. Considering the filtered output sensitivity, the controller design problem can be written as:

$$
\min _{K}\left\|W_{L} S W_{R}\right\|_{\infty}
$$

where $S=(I+G K)^{-1}$ is the output sensitivity function, $W_{L}$ and $W_{R}$ are the left and right weighting filters, where $W_{R}$ is assumed to be invertible. The infinity-norm constraint can be converted to a spectral norm and be approximated with a finite number $N$ of frequencies in $\Omega_{N}$ such that:

$$
\Omega_{N} \subset \Omega=\left\{\omega \mid-\frac{2 \pi}{T_{s}}<\omega<\frac{2 \pi}{T_{s}}\right\} \backslash B_{g} \backslash B_{y}
$$

where $B_{g}$ and $B_{y}$ are respectively the set of finite frequencies in which $G$ and $Y$ are unbounded. It can be shown that the problem in (1) can be written as:

$$
\begin{gathered}
\min _{X, Y, \gamma} \gamma \\
\text { s.t. }\left(W_{L k} S_{k} W_{R k}\right)^{*}\left(W_{L k} S_{k} W_{R k}\right) \leq \gamma I
\end{gathered}
$$

for $k=1, \ldots, N$, where $S_{k}:=\left.S\left(e^{j \omega}\right)\right|_{\omega=\omega_{k}}$. The other variables with subscript $k$ are defined similarly. Replacing $K$ with $X Y^{-1}$ and defining $E=Y-G X$, (2), one obtains:

$$
\left(W_{L k} Y_{k}\right)^{*} \gamma^{-1} W_{L k} Y_{k}-\left(W_{R}{ }^{-1} E_{k}\right)^{*} W_{R_{k}}{ }^{-1} E_{k} \leq 0
$$

Using Schur complement lemma, (3) can be written as:

$$
\left[\begin{array}{cc}
\gamma I & W_{L k} Y_{k} \\
\left(W_{L k} Y_{k}\right)^{*} & \left(W_{R_{k}}^{-1} E_{k}\right)^{*}\left(W_{R_{k}}^{-1} E_{k}\right)
\end{array}\right] \geq 0
$$

The quadratic term can be linearized around an initial stabilizing controller $K_{c}=X_{c} Y_{c}^{-1}$ [17] as follows:

$$
\left(W_{R_{k}}^{-1} E_{k}\right)^{*}\left(W_{R_{k}}^{-1} E_{k}\right) \geq F_{k}
$$

where

$$
\begin{aligned}
F_{k} & =\left(W_{R_{k}}^{-1} E_{k}\right)^{*} W_{R_{k}}^{-1} E_{c k}+\left(W_{R_{k}}^{-1} E_{c k}\right)^{*}\left(W_{R_{k}}^{-1} E_{k}\right) \\
& -\left(W_{R_{k}}^{-1} E_{c k}\right)^{*}\left(W_{R_{k}}^{-1} E_{c k}\right)
\end{aligned}
$$

and $E_{c}=Y_{c}+G X_{c}$. Then, the problem (1) can be represented by the following convex optimization problem:

$$
\begin{aligned}
& \min _{X, Y, \gamma} \gamma \\
& \text { s.t. }\left[\begin{array}{cc}
\gamma I & W_{L k} Y_{k} \\
\left(W_{L k} Y_{k}\right)^{*} & F_{k}
\end{array}\right] \geq 0, \text { for } k=1, \ldots, N
\end{aligned}
$$

For limiting the impact of disturbances on control signals, the input sensitivity function can be limited by defining a constraint on $U=K(I+G K)^{-1}$ as:

$$
\left\|W_{U} U\right\|_{\infty}<1
$$

where $W_{U}$ is the weighting filter corresponding the input sensitivity function. Similar to the previous part, this constraint can be written as:

$$
\left[\begin{array}{cc}
I & W_{U k} X_{k} \\
\left(W_{U k} X_{k}\right)^{*} & E_{k}^{*} E_{c k}+E_{c k}^{*} E_{k}-E_{c k}^{*} E_{c k}
\end{array}\right] \geq 0,
$$

for $k=1, \ldots, N$.

\section{Stability}

In [17], it has been proved that the closed-loop system with the controller $K=X Y^{-1}$ and the plant model $G$ is stable if:

1) the initial controller $K_{c}=X_{c} Y_{c}^{-1}$ is stabilizing,

2) $E^{*} E_{c}+E_{c}^{*} E>0, \forall \omega \in \Omega$

3) $\operatorname{det}\left(Y_{c}\right) \neq 0$ and $\operatorname{det}(Y) \neq 0 \forall \omega \in \Omega$

4) $\operatorname{det}(Y)=\operatorname{det}\left(Y_{c}\right)=0 \forall \omega \in B_{y}$

5) the order of $\operatorname{det}\left(Y_{c}\right)=$ the order of $\operatorname{det}(Y)$

It should be mentioned that Condition 2 is always satisfied because it appears in the infinity-norm constraints. However, Condition 3 should be met by imposing $Y^{*} Y>0$. This can be achieved using the following LMI:

$$
Y^{*} Y_{c}+Y_{c}^{*} Y-Y_{c}^{*} Y_{c}>0, \quad \forall \omega \in \Omega
$$




\section{Reactive Power Sharing Problem}

The objective of the control system of a microgrid is to supply power to loads while keeping voltage magnitude within standard bounds and voltage angle bounded in order to keep synchronism. A general structure of the control system for a microgrid is shown in Fig. 1. This structure is related to an islanded microgrid including synchronous generator, battery storage and PV units. In this structure, $G_{\mathrm{PV}}^{v}, G_{\mathrm{B}}^{v}$ and $G_{\mathrm{S}}^{v}$ are the transfer functions from voltage reference of PV inverter, battery inverter and synchronous generator (i.e. $\bar{v}_{\mathrm{PV}}$, $\bar{v}_{\mathrm{B}}$, and $\bar{v}_{\mathrm{S}}$ ) to the corresponding output voltages (i.e. $v_{\mathrm{PV}}, v_{\mathrm{B}}$, and $v_{\mathrm{S}}$ ). Similarly, $G_{\mathrm{PV}}^{\theta}, G_{\mathrm{B}}^{\theta}$ and $G_{\mathrm{S}}^{\theta}$ are the transfer functions from voltage angle references of PV inverter, battery inverter and synchronous generator (i.e. $\bar{\theta}_{\mathrm{PV}}, \bar{\theta}_{\mathrm{B}}$, and $\bar{\theta}_{\mathrm{S}}$ ) to the corresponding voltage angles (integral of electrical frequency) (i.e. $\theta_{\mathrm{PV}}, \theta_{\mathrm{B}}$, and $\left.\theta_{\mathrm{S}}\right) . G_{g}$ is the transfer function from nodal voltage magnitude and angle at different buses to the active and reactive power injected into the grid. The parametric models related to these transfer functions are developed in [18]. $K_{d}$ is the droop controller for synchronous generator and battery and $K_{S}$ represents the synchronous generator internal speed controller. $G_{L D}$ is the disturbance transfer function from the load powers (i.e. $\left[P_{L}, P_{Q}\right]^{T}$ ) to the output powers of $G_{g}$. The active and reactive power references for battery and synchronous generator (i.e. $\left[\bar{P}_{B}, \bar{P}_{S}, \bar{Q}_{B}, \bar{Q}_{B}\right]$ ) are usually generated by higher level optimization algorithms (e.g. Optimal Power Flow (OPF)), which is not in the scope of this paper. The references of active power for PV buses (i.e. $\bar{P}_{\mathrm{PV}}$ ) are related to solar irradiation and the level of the dc-link voltage, which have high fluctuations. The reactive power references of the PV units (i.e. $\bar{Q}_{\mathrm{PV}}$ ) may be selected based on the outcome of OPF, which are usually updated every few minutes. Controller $K$ is responsible for setting the electrical angles and voltage magnitudes so that the reactive power of the loads are shared proportionally among different PV units.

\section{A. Reactive Power Sharing Formulation}

When the reactive power consumption/injection of the loads are changed, it should be shared among different PV units. Based on the structure mentioned in the previous section for a power grid with $n_{P V}-\mathrm{PV}$ units one can write:

$$
\left[\bar{v}_{\mathrm{PV}}, \bar{\theta}_{\mathrm{PV}}\right]^{T}=K\left[P_{\mathrm{e}}, Q_{\mathrm{e}}\right]^{T}
$$

where $\left[P_{\mathrm{e}}, Q_{\mathrm{e}}\right]^{T}=\left[\bar{P}_{\mathrm{PV}}, \bar{Q}_{\mathrm{PV}}\right]^{T}-\left[P_{\mathrm{PV}}, Q_{\mathrm{PV}}\right]^{T}$ and each variable is a $n_{\mathrm{PV}} \times 1$ vector. (e.g. $\bar{P}_{\mathrm{PV}}=\left[\bar{P}_{\mathrm{PV}}^{1}, \cdots, \bar{P}_{\mathrm{PV}}^{n_{\mathrm{PV}}}\right]^{T}$ and the other variables are defined similarly). The input and output of $U$ are given as:

$$
\left[\bar{v}_{\mathrm{d}}, \bar{\theta}_{\mathrm{PV}}\right]^{T}=U\left[P_{\mathrm{d}}, Q_{\mathrm{d}}\right]^{T}
$$

where $\left[P_{\mathrm{d}}, Q_{\mathrm{d}}\right]^{T}=\left[P_{\mathrm{d}}^{1}, \cdots, P_{\mathrm{d}}^{n_{\mathrm{PV}}}, Q_{\mathrm{d}}^{1}, \cdots, Q_{\mathrm{d}}^{n_{\mathrm{PV}}}\right]^{T}$. Similarly, for the output sensitivity function one can write:

$$
\left[P_{\mathrm{e}}, Q_{\mathrm{e}}\right]^{T}=S\left[P_{\mathrm{d}}, Q_{\mathrm{d}}\right]^{T}
$$

In order to share the reactive power disturbance among different PV units, each unit should have an error with

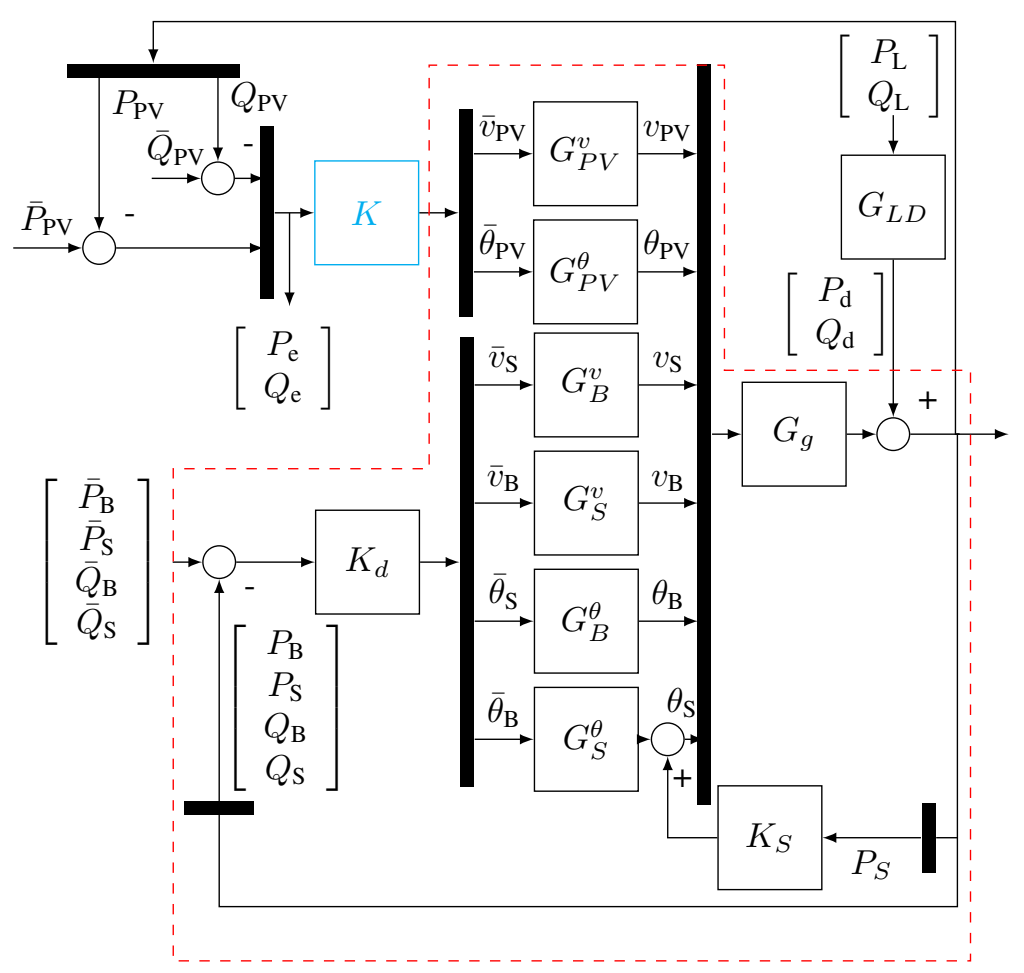

Fig. 1. General structure of the microgrid control system

respect to its reference to compensate the reactive power mismatch until the next output command of OPF. In order to have the impact of reactive power disturbance on reactive power error, one can write

$$
\left[0_{n_{\mathrm{PV}}}, Q_{\mathrm{e}}\right]^{T}=W_{L}^{Q} S W_{R}^{Q}\left[P_{\mathrm{d}}, Q_{\mathrm{d}}\right]^{T}
$$

where $W_{L}^{Q}=\operatorname{diag}\left\{0_{n_{\mathrm{PV}}}, I_{n_{\mathrm{PV}}}\right\}$ and $W_{R}^{Q}=\operatorname{diag}\left\{0_{n_{\mathrm{PV}}}, I_{n_{\mathrm{PV}}}\right\}$. The sharing can be based on any arbitrary preference of the controller designer. We assume that the PV units connected to one microgrid are close to each other and have almost the same per unit spare capacity. Consequently, sharing the reactive power proportional to nominal power can be a reasonable choice. It can be shown that if the following condition holds, the steady-state nominal reactive power sharing can be achieved:

$$
\left.W_{L}^{Q} S W_{R}^{Q}\right|_{\omega=0}=S_{\mathrm{RPS}}
$$

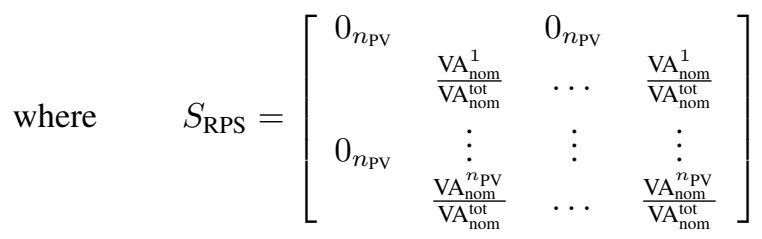

and $\mathrm{VA}^{1}$ is the nominal apparent power of $i$-th PV unit and $\mathrm{VA}_{\mathrm{nom}}^{\mathrm{tot}}=\sum_{i=1}^{i=n_{\mathrm{PV}}} \mathrm{VA}^{i}$.

\section{B. Controller Design Problem for Reactive Power Sharing}

Based on the method described in Section II the controller design problem can be defined as:

$$
\min _{K}\left|W_{L}^{Q} S\left(e^{j \omega_{1}}\right) W_{R}^{Q}-S_{\mathrm{RPS}}\right|
$$


where $\omega_{1}=0$. In order to add tracking and disturbance rejection to the controller, the problem can be written as:

$$
\left\|W_{S} S\right\|_{\infty}<1
$$

For limiting the impact of disturbance on voltage and angle, the following constraint can be added:

$$
\left\|W_{U} U\right\|_{\infty}<1
$$

Using (6) and (8) and considering (16-12), the problem can be written as:

$$
\begin{gathered}
\min _{X, Y, \gamma} \gamma \\
\text { s.t. }\left[\begin{array}{cc}
\gamma I & W_{L}^{Q} Y_{1} \\
\left(W_{L}^{Q} Y_{1}\right)^{*} & F_{1}
\end{array}\right] \geq 0 \\
\\
{\left[\begin{array}{cc}
I & W_{S k} Y_{k} \\
\left(W_{S k} Y_{k}\right)^{*} & E_{k}^{*} E_{c k}+E_{c k}^{*} E_{k}-E_{c k}^{*} E_{c k}
\end{array}\right] \geq 0} \\
{\left[\begin{array}{cc}
I & W_{U k} X_{k} \\
\left(W_{U k} X_{k}\right)^{*} & E_{k}^{*} E_{c k}+E_{c k}^{*} E_{k}-E_{c k}^{*} E_{c k}
\end{array}\right] \geq 0} \\
Y_{k}^{*} Y_{c, k}+Y_{c, k}^{*} Y_{k}-Y_{c, k}^{*} Y_{c, k}>0, \quad \forall\left\{k \mid \omega_{k} \in \Omega_{N}\right\}
\end{gathered}
$$

where

$$
\begin{aligned}
F_{1}= & \left(\left(W_{R}^{Q}\right)^{-1} E_{1}\right)^{*}\left(W_{R}^{Q}\right)^{-1} E_{c k} \\
& +\left(\left(W_{R}^{Q}\right)^{-1} E_{c 1}\right)^{*}\left(\left(W_{R}^{Q}\right)^{-1} E_{1}\right) \\
& -\left(\left(W_{R}^{Q}\right)^{-1} E_{c 1}\right)^{*}\left(\left(W_{R}^{Q}\right)^{-1} E_{c 1}\right)
\end{aligned}
$$

and $W_{L}^{Q}=\operatorname{diag}\left\{0_{n_{\mathrm{PV}}}, I_{n_{\mathrm{PV}}}\right\}$ and $W_{R}^{Q}=\operatorname{diag}\left\{\beta I_{n_{\mathrm{PV}}}, I_{n_{\mathrm{PV}}}\right\}$. $\beta$ is relatively small scaler used to make $W_{R}^{Q}$ invertible.

\section{CAse Study}

In order to validate the performance of the proposed method, it is applied on a case study microgrid through simulation via SimPower of Matlab Simulink. The inverters are modelled using switching elements driven by PWM. The single line diagram of the case study microgrid is shown in Fig. 2. This power distribution grid is composed of a synchronous generator, a battery energy storage unit and three PV units connected to different buses of the grid. The parameters of the grid are mentioned in Table I.

The $X / R$-ratio of different feeders in the grid are different and there is no assumption of dominantly resistive or inductive lines. In this case study, first the frequency response of the system is extracted from the measurements without using the parameters of the grid. Afterwards, the controller is designed and finally, the results are shown.

\section{A. Measurements}

\begin{tabular}{|c|c|}
\hline \multicolumn{2}{|l|}{ Feeders } \\
\hline $\begin{array}{l}\text { Line between bus \#1 and bus \#2 } \\
\text { Line between bus \#2 and bus \#3 } \\
\text { Line between bus \#3 and bus \#4 } \\
\text { Line between bus \#4 and bus \#5 } \\
\text { Line between bus \#6 and bus \#7 } \\
\text { Line between bus \#7 and bus \#8 } \\
\text { Line between bus \#1 and bus \#6 } \\
\text { Line between bus \#2 and bus \#7 }\end{array}$ & $\begin{array}{l}R=0.3 \Omega, X=0.22 \Omega \\
R=0.018 \Omega, X=0.0034 \Omega \\
R=0.018 \Omega, X=0.0034 \Omega \\
R=0.15 \Omega, X=0.11 \Omega \\
R=0.09 \Omega, \quad X=0.017 \Omega \\
R=0.09 \Omega, \quad X=0.017 \Omega \\
R=0.3 \Omega, \quad X=0.22 \Omega \\
R=0.45 \Omega, \quad X=0.085 \Omega\end{array}$ \\
\hline BESS & \\
\hline $\begin{array}{l}\text { Bus \#: } \\
\text { Output filter Parameters: } \\
\text { Time Constants: } \\
\text { Nom. apparent power: }\end{array}$ & $\begin{array}{l}8 \\
R_{t}=10 \mathrm{~m} \Omega, L_{t}=450 \mu \mathrm{H} \\
R_{g}=58 \mathrm{~m} \Omega, L_{g}=420 \mu \mathrm{H}, C_{f}=50 \mu \mathrm{F} \\
\tau_{\omega}=5 \cdot 10^{-4}, \tau_{U}=5 \cdot 10^{-4} \\
30 \mathrm{KVA}\end{array}$ \\
\hline $\begin{array}{l}\text { Synchronous Generator } \\
\text { Bus \#: } \\
\text { Inertia Constant: } \\
\text { Internal Impedance: } \\
\text { Time Constants: } \\
\text { Speed Controller: } \\
\text { Nom. apparent power: }\end{array}$ & $\begin{array}{l}1 \\
\mathrm{H}=1.5 \\
R_{o}=19 \mathrm{~m} \Omega, L_{o}=2.7 \mathrm{mH} \\
\tau_{m}=0.1, \tau_{U}=0.05 \\
k_{p}=3.18, k_{i}=4.77, k_{d}=0.8, T_{f}=0.05 \\
45 \mathrm{KVA}\end{array}$ \\
\hline $\begin{array}{l}\text { Inverter } \\
\text { Bus \#: } \\
\text { Switching Frequency: } \\
\text { DC Voltage: }\end{array}$ & $\begin{array}{l}3,4,5,8 \\
15 \mathrm{KHz} \\
325 \mathrm{~V}\end{array}$ \\
\hline $\begin{array}{l}\text { PV } \\
\text { Bus \#: } \\
\text { Output filter Parameters: } \\
\text { Nominal Power: }\end{array}$ & $\begin{array}{l}3,4,5 \\
R_{t}=10 \mathrm{~m} \Omega, L_{t}=450 \mu \mathrm{H} \\
R_{g}=58 \mathrm{~m} \Omega, L_{g}=420 \mu \mathrm{H}, C_{f}=50 \mu \mathrm{F} \\
{[30,20,40] \mathrm{kW}}\end{array}$ \\
\hline $\begin{array}{l}\text { Loads } \\
\text { Bus \#: } \\
\text { Active/Reactive Power: }\end{array}$ & $\begin{array}{l}3,6,7 \\
{[30,20,25] \mathrm{kW} /[0,0,0] \mathrm{VAr}}\end{array}$ \\
\hline
\end{tabular}

An external excitation can be added to the inputs of the plant in order to find the frequency response of the system. In this case study, a multi-period PRBS signal with an amplitude of 0.01p.u. has been added to $\bar{v}_{\mathrm{PV}}^{1}, \bar{v}_{\mathrm{PV}}^{2}$, and $\bar{v}_{\mathrm{PV}}^{3}$ and a PRBS signal with amplitude of 0.002 p.u. to $\bar{\theta}_{\mathrm{PV}}^{1}, \bar{\theta}_{\mathrm{PV}}^{2}$, and $\bar{\theta}_{\mathrm{PV}}^{3}$ during 6 separate experiments. During each experiment, $\left[P_{\mathrm{PV}}, Q_{\mathrm{PV}}\right]^{T}$ in p.u. are sampled with a
TABLE I

CAse Study GRID PARAmeters

frequency of $100 \mathrm{~Hz}$. Taking the Fourier transform of the measured data, $G(j \omega) \in \mathbb{C}^{6 \times 6}$ is calculated. In this case study, the frequency points are linearly distributed between 0 and $50 \mathrm{~Hz}$. The order of PRBS signal is 7 (which is equivalent to 127 sample per period) and 5 periods has been applied. Consequently, the total duration of measurement is 38.1 s $(=127$ (samples per periods $) \times 5$ (periods $) \times$ $10^{-2}$ (sampling time $) \times 6$ (inputs) $)$. As an example, measured data corresponding to $Q_{\mathrm{PV}}^{3}$ while adding last four periods of PRBS signal to $\bar{\theta}_{\mathrm{PV}}^{1}$ and the resulting frequency response are shown in Fig. 3. The frequency response used for the controller design is the average of frequency responses of different periods at each frequency points.

\section{B. Controller Design}

The convex optimization problem in (18) has been solved based on the frequency response of the system using the measured data. An 8th order stabilizing initial controller with a very small gain is chosen:

$$
Y_{c}=z^{8} I_{6}, X_{c}=\epsilon z^{8} I_{6}, K_{c}=X_{c} Y_{c}^{-1}
$$

where $\epsilon=0.05$. It should be noted that $z^{8}$ term in both $Y_{c}$ and $X_{c}$ has been added to satisfy the fourth stability condition.

The inverse of the weighing filter for output sensitivity function $W_{S_{k}}^{-1}$ for $\omega_{k} \in \Omega_{N}$ is selected as $\operatorname{diag}\left(5 \mathrm{~dB} I_{3}, I_{3}\right)$ for $\omega_{k}<B W$ where $B W=15 \mathrm{rad} / \mathrm{s}$ is the desired closed-loop bandwidth. For high frequencies, $\omega_{k}>B W$, the inverse of the weighting filter is chosen as $6 \mathrm{~dB} I_{6}$ to limit 


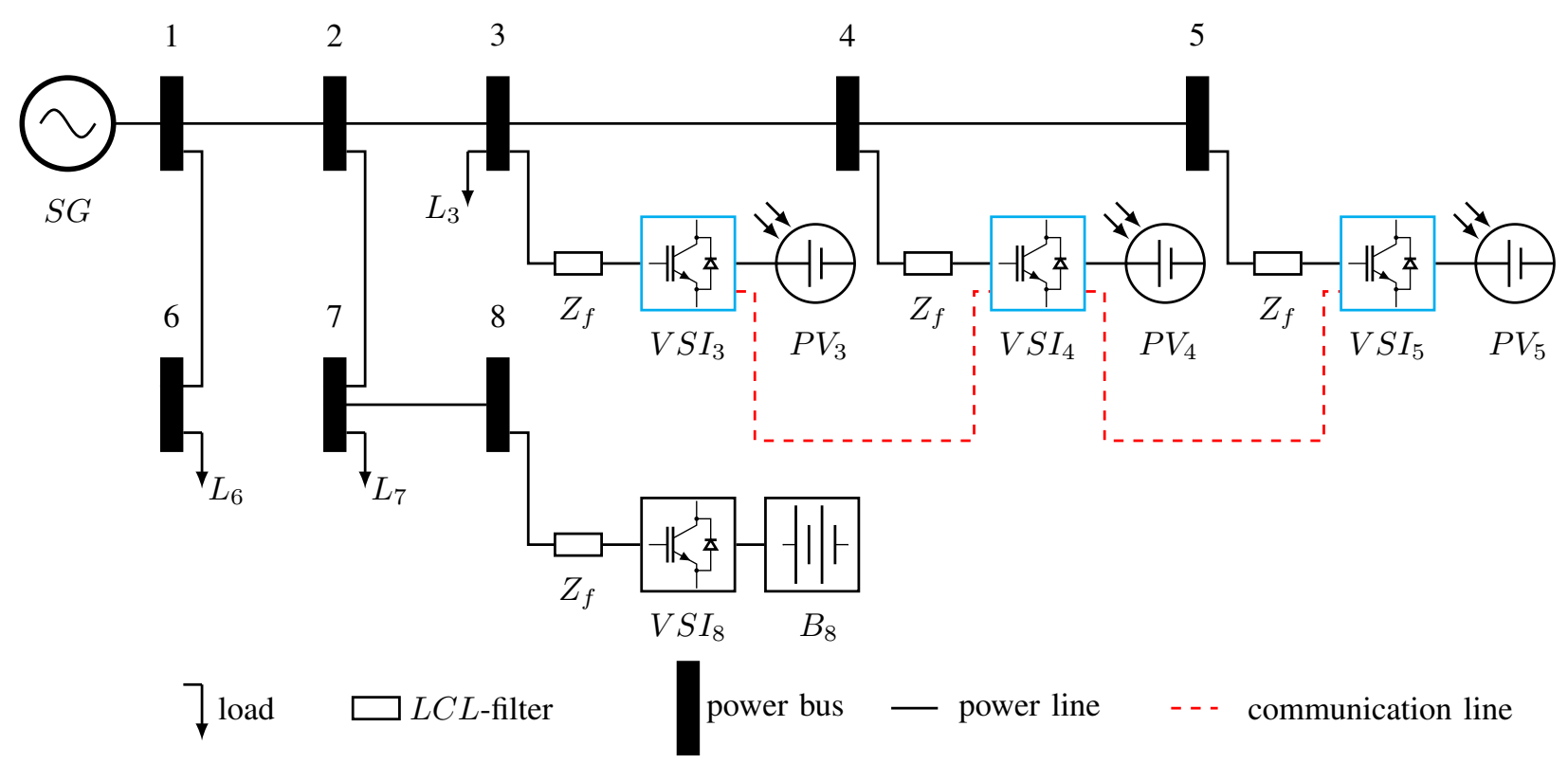

Fig. 2. Single line diagram of case study micro grid
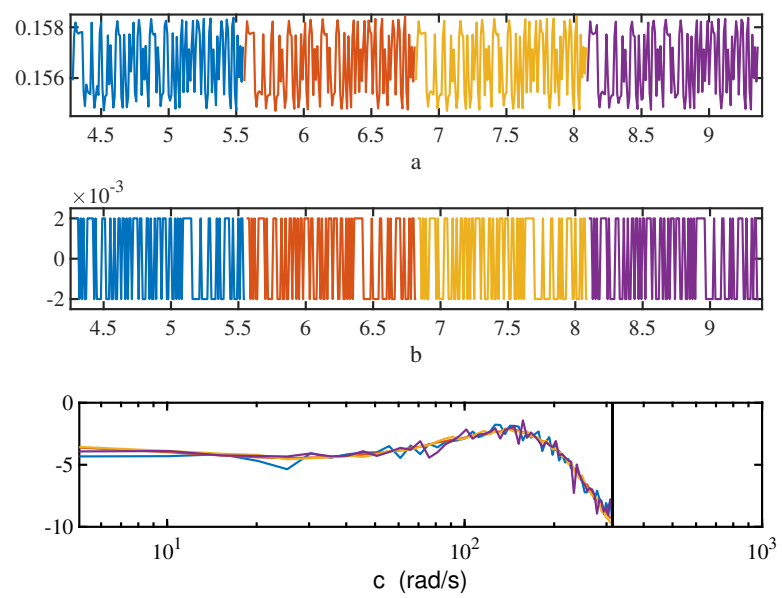

Fig. 3. a) measured data corresponding to $Q_{\mathrm{PV}}^{3}$, b) last four periods of PRBS signal to $\bar{\theta}_{\mathrm{PV}}^{1} \mathrm{c}$ ) the corresponding frequency response

the maximum singular value of $S$ to $6 \mathrm{~dB}$ and obtaining a good stability margin. In order to limit the impact of high frequency harmonics on voltages and angles, the inverse of the weighting filter for input sensitivity function $W_{U_{k}}^{-1}$ is selected as $20 \mathrm{~dB} I_{6}$ for $\omega_{k}<7 \times B W$ and $I_{6}$ for $\omega_{k}>$ $7 \times B W$.

\section{Distributed Structure}

As shown in Fig. 2, the system in the case study has distributed control structure. In this system, the data can be transferred between controllers of bus number 3 and bus number 4 as well as between 4 and 5 but there exists no data link between the inverters at bus number 3 and bus number 5 . This structure is considered in the controller design process by setting the corresponding parameters to zero where there
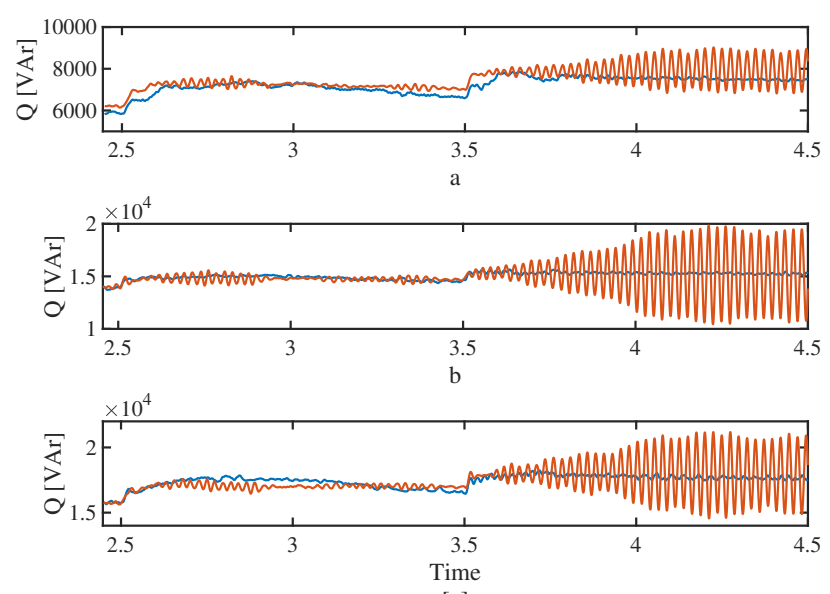

$[c]$

Fig. 4. Reactive power of three PV units, blue: proposed method, red: primary droop and secondary central integrator, a) reactive power of PV number $1, \mathrm{~b})$ reactive power of $\mathrm{PV}$ number 2 , c) reactive power of $\mathrm{PV}$ number 3

is no communication link.

\section{Results}

The performance of the designed controller has been validated through simulation using Matlab Simulink and are compared with conventional droop control as primary control combined with a central integrator as the secondary controller. To test the reactive power sharing performance, a 0.1 p.u. reactive load has been added to bus number 6 at $\mathrm{t}=2.5 \mathrm{~s}$ and another 0.1 p.u. reactive load has been added to bus number 3 at $\mathrm{t}=3.5 \mathrm{~s}$. The reactive powers of the three PV units are shown in Fig. 4. It can be seen that the reactive powers have been shared based on their nominal apparent 

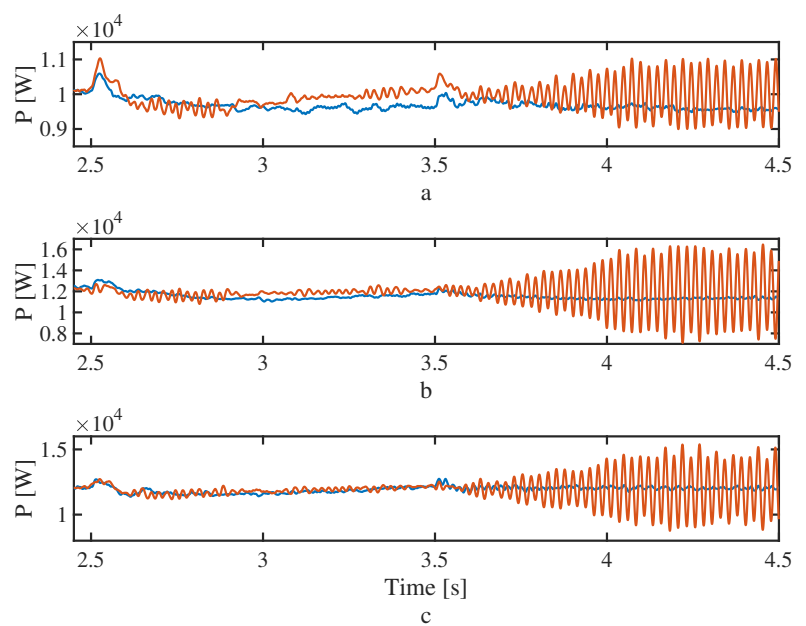

Fig. 5. Active power of three PV units. blue: proposed method, red: primary droop and secondary central integrator, a) active power of PV number $1, b$ ) active power of PV number 2, c) active power of PV number 3
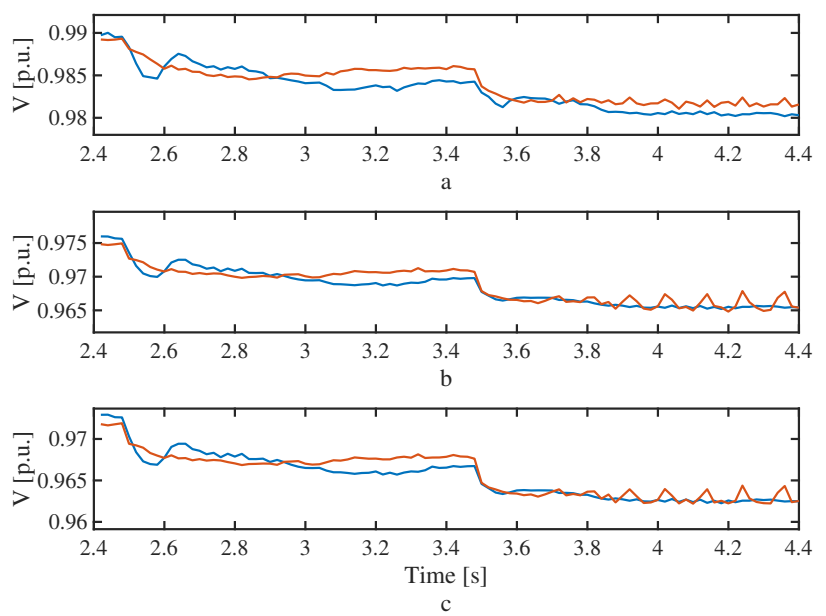

Fig. 6. RMS p.u. voltage of three PV units, blue: proposed method, red: primary droop and secondary central integrator, a) voltage of PV number $1, \mathrm{~b})$ voltage of PV number $2, \mathrm{c})$ voltage of PV number 3

powers. As mentioned earlier, the droop control does not guarantee stability and it can be seen in this case study that the droop control fails to control reactive power after adding the second load to the system. The active powers of three PV after load disturbance are shown in Fig. 5. As shown in the figure, the controller rejects the disturbance on active power which leads to less stress of DC-link while the droop controller has a higher peak in active power and oscillatory mode after adding the second load. The RMS voltages of PV buses are shown in Fig. 6. As shown in this figure, voltages of PV units are kept within the standard band while the droop controller shows high fluctuations in voltage.

\section{CONCLUSIONS}

In this paper, a data-driven controller design approach has been proposed in order to employ the spare capacity of
PV units in reactive power sharing. In this method, there is no need for a parametric model of the power system which is usually a problem in controller design in power systems. Instead, the measurement data has been used in the controller design process. In this method, there is no assumption on power feeders impedance such as dominantly inductive or dominantly resistive, which limits the generality of other methods. The proposed method can be applied to different control structures, i.e. centralized, distributed and decentralized during control design. The performance of the proposed method has been validated through simulation in a three-phase microgrid including synchronous generator, storage systems and PV units. The results show that this method can share reactive power among PV units while providing disturbance rejection in active power and voltage.

\section{REFERENCES}

[1] J. Liang and K. Zhu, "Coded switching scheme for monitoring the operation of distribution capacitors," IEEE Transactions on Power Delivery, vol. 33, no. 6, pp. 3075-3084, 2018.

[2] K. Diehl, J. A. Diaz de Leon, and M. Ghorai, "Applying svcs on distribution systems," in IEEE PES T D 2010, 2010, pp. 1-7.

[3] D. B. Kulkarni and G. R. Udupi, "Ann-based svc switching at distribution level for minimal-injected harmonics," IEEE Transactions on Power Delivery, vol. 25, no. 3, pp. 1978-1985, 2010.

[4] Jen-Hung Chen, Wei-Jen Lee, and Mo-Shing Chen, "Using a static var compensator to balance a distribution system," IEEE Transactions on Industry Applications, vol. 35, no. 2, pp. 298-304, 1999.

[5] S. Ziaeinejad and A. Mehrizi-Sani, "Design tradeoffs in selection of the dc-side voltage for a d-statcom," IEEE Transactions on Power Delivery, vol. 33, no. 6, pp. 3230-3232, 2018.

[6] C. Wang, X. Yin, Z. Zhang, and M. Wen, "A novel compensation technology of static synchronous compensator integrated with distribution transformer," IEEE Transactions on Power Delivery, vol. 28, no. 2, pp. 1032-1039, 2013.

[7] S. Li, Y. Li, Y. Cao, Y. Tan, and B. Keune, "Capacity optimisation method of distribution static synchronous compensator considering the risk of voltage sag in high-voltage distribution networks," IET Generation, Transmission Distribution, vol. 9, no. 16, pp. 2602-2610, 2015.

[8] S. Jain, M. B. Shadmand, and R. S. Balog, "Decoupled active and reactive power predictive control for pv applications using a gridtied quasi-z-source inverter," IEEE Journal of Emerging and Selected Topics in Power Electronics, vol. 6, no. 4, pp. 1769-1782, 2018.

[9] A. Micallef, M. Apap, C. Spiteri-Staines, J. M. Guerrero, and J. C. Vasquez, "Reactive power sharing and voltage harmonic distortion compensation of droop controlled single phase islanded microgrids," IEEE Transactions on Smart Grid, vol. 5, no. 3, pp. 1149-1158, 2014.

[10] H. Han, Y. Liu, Y. Sun, M. Su, and J. M. Guerrero, "An improved droop control strategy for reactive power sharing in islanded microgrid," IEEE Transactions on Power Electronics, vol. 30, no. 6, pp. 3133-3141, 2015.

[11] F. Valenciaga and C. A. Evangelista, "2-sliding active and reactive power control of a wind energy conversion system," IET Control Theory Applications, vol. 4, no. 11, pp. 2479-2490, 2010.

[12] S. Bolognani, R. Carli, G. Cavraro, and S. Zampieri, "Distributed reactive power feedback control for voltage regulation and loss minimization," IEEE Transactions on Automatic Control, vol. 60, no. 4, pp. 966-981, 2015.

[13] M. P. S. Gryning, Q. Wu, Kocewiak, H. H. Niemann, K. P. H. Andersen, and M. Blanke, "Stability boundaries for offshore wind park distributed voltage control," IEEE Transactions on Control Systems Technology, vol. 25, no. 4, pp. 1496-1504, 2017.

[14] W. Meng, Q. Yang, and Y. Sun, "Guaranteed performance control of dfig variable-speed wind turbines," IEEE Transactions on Control Systems Technology, vol. 24, no. 6, pp. 2215-2223, 2016.

[15] Y. Fan, G. Hu, and M. Egerstedt, "Distributed reactive power sharing control for microgrids with event-triggered communication," IEEE Transactions on Control Systems Technology, vol. 25, no. 1, pp. 118128, 2017. 
[16] M. Todescato, J. W. Simpson-Porco, F. Dörfler, R. Carli, and F. Bullo, "Online distributed voltage stress minimization by optimal feedback reactive power control," IEEE Transactions on Control of Network Systems, vol. 5, no. 3, pp. 1467-1478, 2018.

[17] A. Karimi and C. Kammer, "A data-driven approach to robust control of multivariable systems by convex optimization," Automatica, vol. 85, pp. 227-233, 2017.

[18] C. Kammer and A. Karimi, "Decentralized and distributed transient control for microgrids," IEEE Transactions on Control Systems Technology, vol. 27, no. 1, pp. 311-322, 2019. 